\title{
A Novel Nanostructured Scanning Near Field Optical Microscopy Probe Based on an Adirectional Asymmetry
}

\author{
Valeria Lotito ${ }^{1,2, *}$, Christian Hafner ${ }^{2}$, Urs Sennhauser ${ }^{1}$, and Gian-Luca Bona ${ }^{1,2}$ \\ ${ }^{1}$ EMPA, Swiss Federal Laboratories for Materials Science and Technology, Electronics/Metrology Laboratory, \\ Ueberlandstrasse 129, CH-8600 Dübendorf, Switzerland \\ ${ }^{2}$ Laboratory of Electromagnetic Fields and Microwave Electronics, ETH Zurich, Gloriastrasse 35, 8092 Zurich, Switzerland
}

\begin{abstract}
Recently a novel class of scanning near field optical microscopy probes based on an adirectional asymmetry (i.e., an asymmetry encompassing all spatial directions) in form of a spiral-like corrugation has been proposed. Although such probes would entail an impressive simplification in experimental procedures as they allow superfocusing for any arbitrarily oriented linearly polarized excitation, their actual fabrication looks much more challenging compared to probes based on directional asymmetries (i.e., asymmetries lying along one specific spatial direction). Here we propose a new simpler implementation of adirectional asymmetry which shares the same advantages as the one based on a spiral nanostructuring of the external metal coating, while being more feasible for fabrication.
\end{abstract}

Keywords: Scanning Near Field Optical Microscopy, Apertureless Optical Probes, Near Field Optics, Probe Geometry.

\section{INTRODUCTION}

The design of efficient scanning near field optical microscopy (SNOM) probes has been a hot research issue over the past years. ${ }^{1,2}$ Near field microscopy is based on the near field interaction between the probe and the sample, which accounts for the role played by the probe itself in the eventually achievable resolution and signal throughput, independently of whether the tip is used for near field excitation of the sample and/or near field collection of the signal from a sample broadly illuminated in the far field. ${ }^{3}$

Although aperture SNOM probes (metal-coated dielectric probes with an aperture opened at the apex) are still the most commonly used tip structure, they do not guarantee adequate resolution and throughput. In fact, in this case resolution is mostly given by the size of the aperture, which cannot be arbitrarily decreased in order to avoid a dip in the signal throughput (the latter cannot be compensated in its turn by an increase in the input power because of the risk of thermal damage). ${ }^{3,4}$ Moreover, as they are typically excited with a linearly polarized mode, this results in an asymmetric near field distribution, a further drawback for this class of probes. ${ }^{5}$ Different routes for optimization have been followed, some of them still based on aperture probes with optimized taper profiles or

${ }^{*}$ Author to whom correspondence should be addressed. aperture shapes, ${ }^{6-10}$ some others on completely new probe concepts like apertureless probes. ${ }^{11-13}$

Apertureless probes can be either fully metal probes or fully metal-coated dielectric probes. Fully metal-probes are usually excited in the far field, resulting in a strong background which can be easily eliminated in fully metalcoated probes under internal back excitation., 12,14 Under properly polarized excitation strong field localization and enhancement are reachable in fully metal-coated dielectric probes. Such behaviour is due to the nanofocusing of the surface plasmon polaritons (SPPs) excited on the metal cladding by the internal waveguide mode: if such SPPs have the same polarity on the opposite sides of the probe, as is the case for those excited by a radially polarized waveguide mode, they interfere constructively at the tip apex because of the axial symmetry of the mode and the probe, generating the desired single hot spot; on the contrary, if they have opposite polarities, as for a linearly polarized waveguide mode, they interfere destructively, resulting in a broad, weak, two-lobed distribution. ${ }^{13,15}$

Superfocusing under linearly polarized excitation can be achieved if the symmetry of the probe is broken. Different modifications have been proposed, like slits and corrugations in the metal coating or oblique cuts in a plane neither orthogonal nor parallel to the probe axis and consisting in the removal of both the dielectric and the metal. ${ }^{16-18}$ The behaviour of tip on aperture probes and 
probes based on a monopole antenna can be also explained with similar considerations. ${ }^{19,20}$ As an alternative, instead of an asymmetric probe structure, an asymmetric illumination scheme has been suggested: in the offset aperture probe, the surface plasmons are excited only on one side of an apertureless probe via an opening close to the probe base, ${ }^{21}$ while asymmetric single-sided SPP excitation results in field localization in an axisymmetric apertureless probe. ${ }^{22}$ In all the previous cases the asymmetry is directional, i.e., it lies along one specific spatial direction. This means that if $z$ is the probe axis, a properly designed asymmetry introduced along $x$ gives rise to superfocusing under $x$ linearly polarized excitation, while no such effect will be observed under $y$ polarized excitation. Although the introduction of the asymmetry entails a significant simplification in the injection procedures compared with an axisymmetric structure (as the need for a cumbersome radially polarized excitation is lifted and superfocusing can be achieved under an easier linearly polarized excitation), it still requires some control on the mutual alignment between the probe and the input polarization direction. $^{23}$

A new class of asymmetric structures has recently been proposed, by extending the asymmetry to all spatial directions. As a proof of concept of this novel adirectional asymmetry, a spiral-like corrugation has been analysed and superfocusing under any arbitrarily oriented linearly polarized excitation has been numerically demonstrated, which would waive any requirement on the alignment. ${ }^{24} \mathrm{How}-$ ever, the actual fabrication of such a corrugation appears extremely challenging, even though spiral patterns have been realized in two-dimensional (2D) structures for plasmonic lenses, ${ }^{25}$ and three-dimensional (3D) helix-shaped metamaterials. $^{26}$

In this paper, we propose a novel implementation of adirectional asymmetries, which appears more feasible for fabrication. We show that the more exotic spiral corrugation can be replaced by a simpler series of azimuthal corrugations over arcs of circumference in planes orthogonal to the probe axis shifted one with respect to the other to
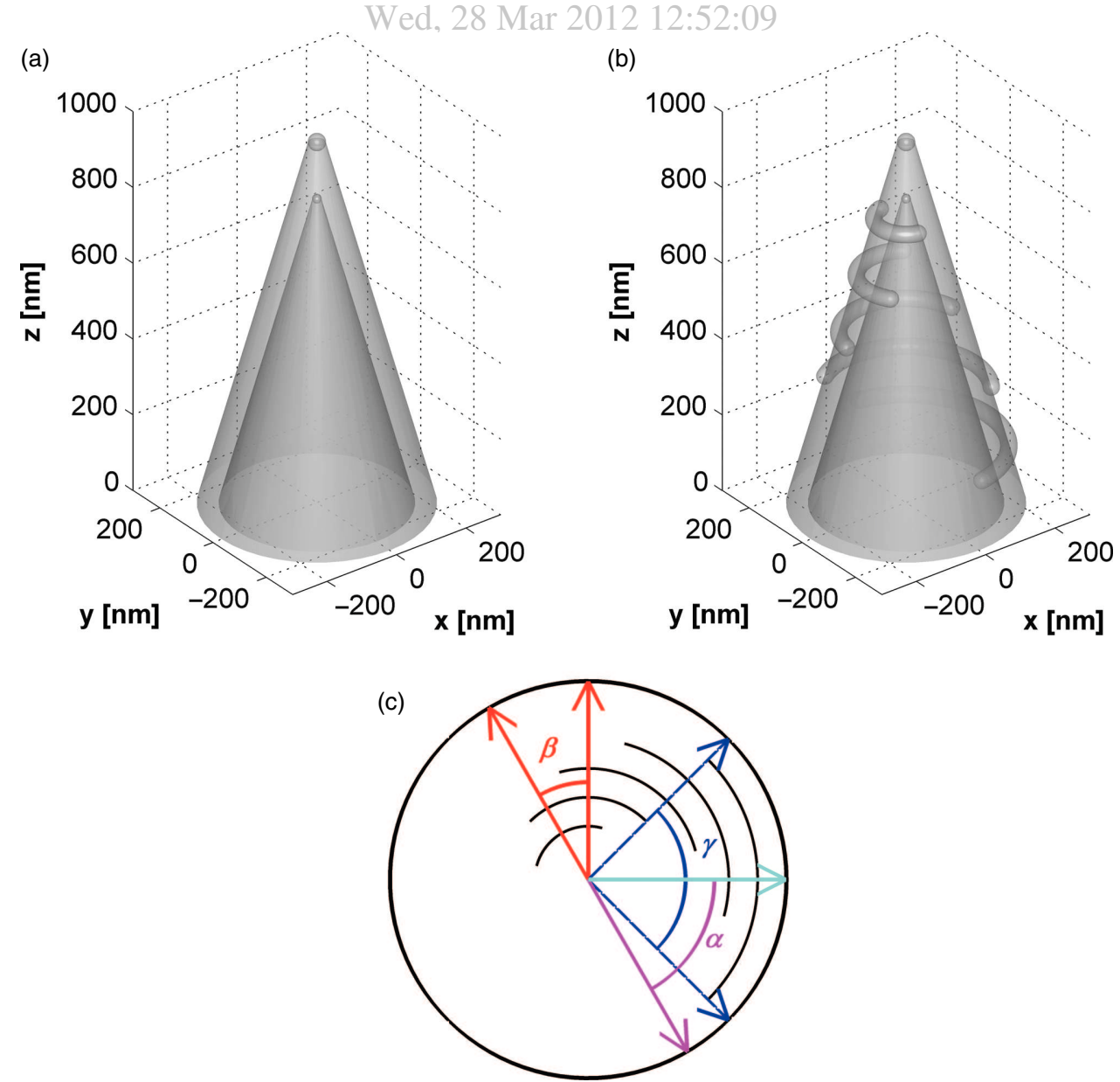

Fig. 1. Sketch of the probe structures: (a) axisymmetric fully metal-coated probe; (b) probe with azimuthal corrugations arranged in a spiral-like fashion; (c) schematics of a 2D projection of the probe with corrugations and relevant parameters; the black arcs represent the corrugations; the angle $\alpha$ is the one between the direction of the input linear polarization (indicated by the magenta line) and the angle bisector of the bottom corrugation (represented with the cyan line); the angle $\beta$ is the shift angle measured between the angle bisectors of two consecutive corrugations, while the angle $\gamma$ is the angular extension of each corrugation. According to Figure 1(c), the angle bisector of the bottom corrugation is along the $x$ axis. 
create a spiral arrangement. The parameters of this new structure can be varied to improve the field localization and enhancement for any orientation of the input linearly polarized excitation.

\section{COMPUTATIONAL MODEL}

Simulations have been performed using a finite element based software, Comsol Multiphysics, as the use of unstructured grids enables to handle complex geometries more easily than other methods like the finite difference time domain method. The need for 3D calculations was dictated by the lack of either rotational symmetry, typical of the axisymmetric structure, or reflection symmetry, as for directional asymmetries which exhibit a plane of symmetry. Hence, the problem could not be tackled with simpler approaches like the body of revolution finite difference time domain method or a $2 \mathrm{D}$ approximation as done in previous works for axisymmetric probe structures. 5,8 The sketches of the fully metal-coated axisymmetric probe and of the new tip based on azimuthal corrugations arranged in a spiral-like fashion are reported in Figures 1(a and b).

Both the probes consist of a silica core $(n=1.5)$ covered by an aluminium coating $(n=0.645+5.029 i$ at the operating wavelength of $532 \mathrm{~nm}$ ). The radii of the inner silica cone and of the outer edge of the metallic hollow cone (both with a $30^{\circ}$ full cone angle) are $225 \mathrm{~nm}$ and $275 \mathrm{~nm}$, respectively. The inner silica cone and the aluminium hollow cone have rounded terminations with radii of 10 and $20 \mathrm{~nm}$, respectively. For the structure based on azimuthal corrugations, we considered as an example five truncated toroids with hemispherical terminations. In order to create a spiral arrangement (and hence an adirectional asymmetry distributed over all spatial directions), the corrugations were shifted one with respect to the other as visible in Figure 1(c). The effect of variations in the shift angle $\beta$ (identical for any two consecutive corrugations) has been examined as well as the impact of variable radius and variable azimuthal extension $\gamma$ of the single corrugations (which means the arc of circumference over which corrugations are spanned). In Figure 1(c), the angle $\alpha$ between the direction of the input linearly polarized excitation and the angle bisector of the bottom corrugation is also defined. According to the sketches in Figures 1(b and c), the angle bisector of the bottom corrugation is along the $x$ axis. The $z$ spacing between consecutive corrugations is $150 \mathrm{~nm}$. Each azimuthal corrugation can be a groove or a bump, i.e., the truncated toroid can be filled either with air or with metal. In a previous study on directional asymmetries based on such azimuthal corrugations (null shift angle $\beta$ between consecutive corrugations), it was shown that the combination resulting in maximum field enhancement consisted in an alternation of grooves and bumps starting from the bottom corrugation and was named amama after the initial of the filling material ( $a$ stands for air and $m$ for metal). ${ }^{17}$ On the grounds of this study we focused our attention on the same structure.

\section{SPIRAL-ARRANGED AZIMUTHAL CORRUGATIONS: DIRECTIONAL VERSUS ADIRECTIONAL ASYMMETRIES}

As pointed out earlier, in order to have an asymmetry distributed along multiple spatial directions, the shift angle $\beta$ should be different from zero. Figure $2(a \div d)$ report the square of the norm of the electric field under $x$ and $y$ linearly polarized excitation for a shift angle of $0^{\circ}$ (directional asymmetry) and for one of $45^{\circ}$ (adirectional asymmetry). Distributions (calculated over a $600 \mathrm{~nm}$ by $600 \mathrm{~nm}$ square area centred at the tip apex in a transverse plane located at $10 \mathrm{~nm}$ from the probe apex) are normalized to the peak value achieved in an axisymmetric probe (Fig. 1(a)) under radially polarized excitation, which represents our reference for comparison. For such axisymmetric probe, broad two-lobed distributions were obtained under linearly polarized excitation, with a peak value about 50 times lower than the one achievable under radially polarized excitation and an average size of $400 \mathrm{~nm}$ against the $38 \mathrm{~nm}$ full width at half maximum (FWHM) of the radial spot.

The total azimuthal extension $\gamma$ for each of the five corrugations amounts to $160^{\circ}$ and the radius of the truncated toroids is $20 \mathrm{~nm}$. In both the cases we considered the angle bisector of the bottom corrugation located along the $x$ axis, as depicted in Figure 1(c), which means that, in case of directional asymmetry $\left(\beta=0^{\circ}\right)$, all the five corrugations create a strong asymmetry along $x$. Moreover, according to the notation illustrated in Figure 1(c), $x$ and $y$ linearly polarized excitation will correspond to $\alpha=0^{\circ}$ and $\alpha=90^{\circ}$ respectively.

As expected, for $\beta=0^{\circ}$, strong field localization is observed under $x$ linearly polarized excitation, with a peak value about 8 times higher than the one attainable in an axisymmetric probe under radially polarized excitation (Fig. 2(a)), while a broad weak two-lobed distribution results from the orthogonal input linear polarization (Fig. 2(b)), as no asymmetry is present along $y$, and no significant change with respect to an axisymmetric probe under linearly polarized excitation is present. On the contrary, for $\beta=45^{\circ}$, superfocusing is achieved for both the orthogonal polarizations (Figs. 2(c and d)) as the asymmetry is effective in both directions. Furthermore, as visible, the two distributions show close resemblance in both peak value and FWHM, though the peak value is lower than the one achieved in case of directional asymmetry under $x$ polarized excitation for both the input polarizations. Note that $\alpha=0^{\circ}$ corresponds to the position of best alignment of the input linear polarization with respect to the asymmetry in case of the directional asymmetry, with $\alpha=90^{\circ}$ representing the maximum misalignment. On the contrary, 

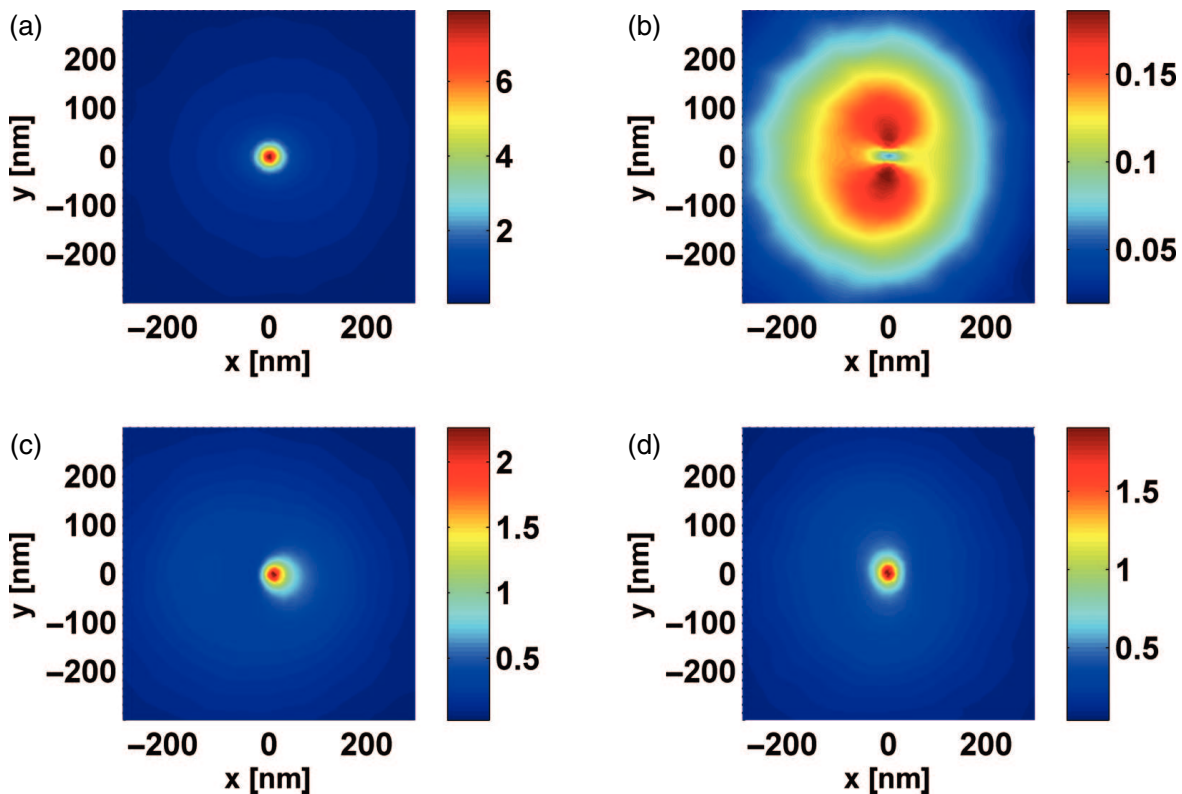

Fig. 2. Comparison between probes with $\beta=0^{\circ}$ (directionab asymmetry) and $\beta=45^{\circ}$ (adirectional asymmetry) $\left(\gamma=160^{\circ}\right.$; radius $20 \mathrm{~nm}$ ): (a) normalized near field distributions in a plane located at $10 \mathrm{~nm}$ from the apex of a probe with $\beta=0^{\circ}$ under $x$ linearly polarized excitation; (b) normalized near field distributions in a plane located at $10 \mathrm{~nm}$ from the apex of a probe with $\beta=0^{\circ}$ under $y$ linearly polarized excitation; (c) normalized near field distributions in a plane located at $10 \mathrm{~nm}$ from the apex of a probe with $\beta=45^{\circ}$ under $x$ linearly polarized excitation; (d) normalized near field distributions in a plane located at $10 \mathrm{~nm}$ from the apex of a probe with $\beta=45^{\circ}$ under $y$ linearly polarized excitation.

for the adirectional asymmetry, different orientations are almost equivalent.

In order to further confirm that the alignment of the input linear polarization along one specific direction is no longer necessary with this novel adirectional asymmetry, we compared the behaviour of the probe with directional asymmetry $\left(\beta=0^{\circ}\right.$; Figs. $3(\mathrm{a}$ and $\left.\mathrm{b})\right)$ with the one based on spiral-arranged corrugations $\left(\beta=45^{\circ}\right.$; (a)

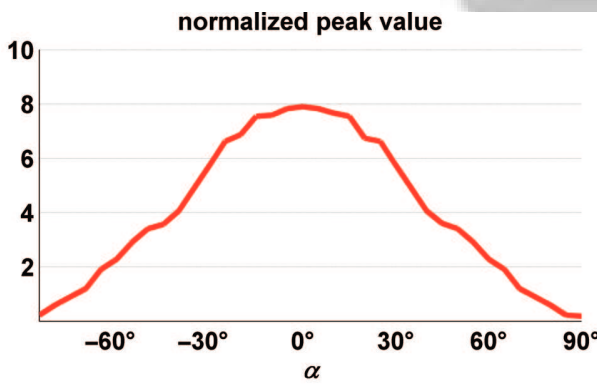

(c)

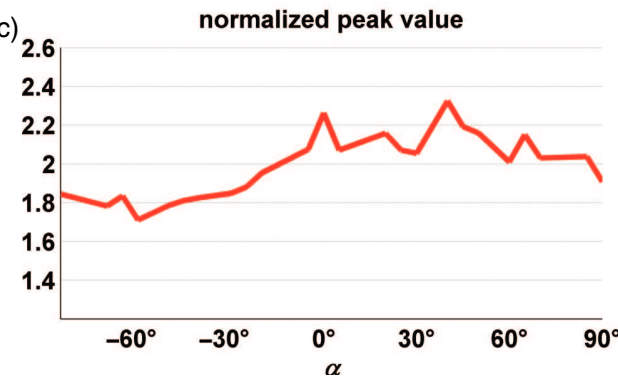

(b)

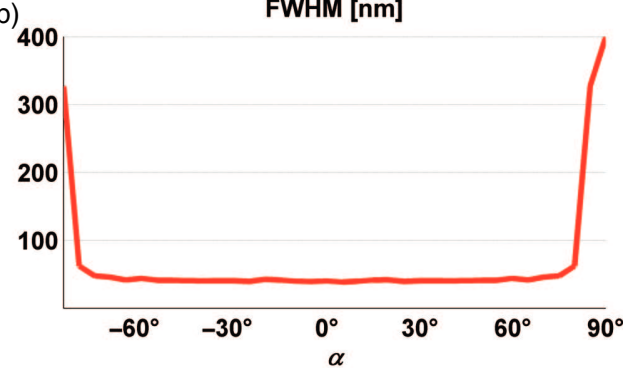

(d)

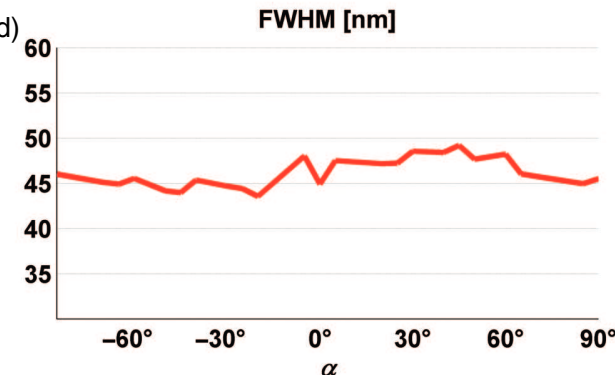

Fig. 3. Comparison between probes with $\beta=0^{\circ}$ (directional asymmetry) and $\beta=45^{\circ}$ (adirectional asymmetry) $\left(\gamma=160^{\circ}\right.$; radius $20 \mathrm{~nm}$ ): (a) peak value of the near field distribution for the probe with $\beta=0^{\circ}$ normalized to the one of the axisymmetric probe under radially polarized excitation for variable orientation $\alpha$ of the corrugations with respect to the input linear polarization; (b) FWHM of the near field distribution for the probe with $\beta=0^{\circ}$ for variable orientation $\alpha$ of the corrugations with respect to the input linear polarization; (c) peak value of the near field distribution for the probe with $\beta=45^{\circ}$ normalized to the one of the axisymmetric probe under radially polarized excitation for variable orientation $\alpha$ of the corrugations with respect to the input linear polarization; (d) FWHM of the near field distribution for the probe with $\beta=45^{\circ}$ for variable orientation $\alpha$ of the corrugations with respect to the input linear polarization. 
Figs. 3(c and d)) under variable orientation of the corrugations with respect to the input linear polarization. More specifically, the FWHM and the peak value normalized to the one of the axisymmetric probe under radially polarized excitation were examined. As explained in the previous paragraph, the mutual orientation is defined as the angle $\alpha$ between the direction of the input linear polarization and the angle bisector of the bottom corrugation; the mutual orientation specified by $\alpha$ was changed from $-85^{\circ}$ to $90^{\circ}$ to encompass all the possible mutual positions. From the graphs, it can be inferred that deviations of the peak value from the average are below $20 \%$ and those of the spot size are less than $10 \%$ for the probe with adirectional asymmetry against deviations of about $100 \%$ and $450 \%$ for the one with directional asymmetry, which demonstrates that the tip with spiral-arranged corrugations can actually afford the achievement of superfocusing under arbitrarily oriented linearly polarized excitation. In particular, the negligible variations in the FWHM indicate that the ultimate achievable resolution is almost insensitive to the input polarization direction. Similar trends were observed by changing the chirality, i.e., the handedness, of the spiral-like arrangement from counter clockwise to clockwise.

In the next paragraph, we will investigate the impact of geometric parameters of the corrugations on the superfocusing effect, in particular the shift angle $\beta$, the angular extension $\gamma$ and the radius of the corrugation.

\section{SPIRAL-ARRANGED AZIMUTHAL CORRUGATIONS: VARIATIONS IN GEOMETRIC PARAMETERS}

In order to study how sensitive such superfocusing effect is to the shift between consecutive corrugations, we carried out a set of simulations with variable shift angle $\beta$. We varied this parameter from $0^{\circ}$ to $45^{\circ}$, with a step of $7.5^{\circ}$. The corresponding total shift between the bottom and top corrugation is $(n-1) \cdot \beta$, where $n$ is the number of the azimuthal corrugations. For each of the simulated structures, we considered the average behaviour over all the possible orientations of the asymmetry with respect to the input linearly polarized mode as specified by $\alpha$, that is, the average normalized peak value and the average FWHM over all the possible mutual orientations of the corrugations with respect to the input linearly polarized excitation (i.e., over all $\alpha$ between $-85^{\circ}$ and $90^{\circ}$ ), together with the maximum variations of these figures of merit from the correspondling averages. Figure 4 illustrates the results obtained for corrugations with angular extension $\gamma$ of $160^{\circ}$ and radius $20 \mathrm{~nm}$.

As the shift angle $\beta$ increases, the average spot size decreases because the asymmetry becomes effective in multiple directions, which allows the achievement of superfocusing under multiple mutual orientations of the input linear polarization with respect to the corrugations
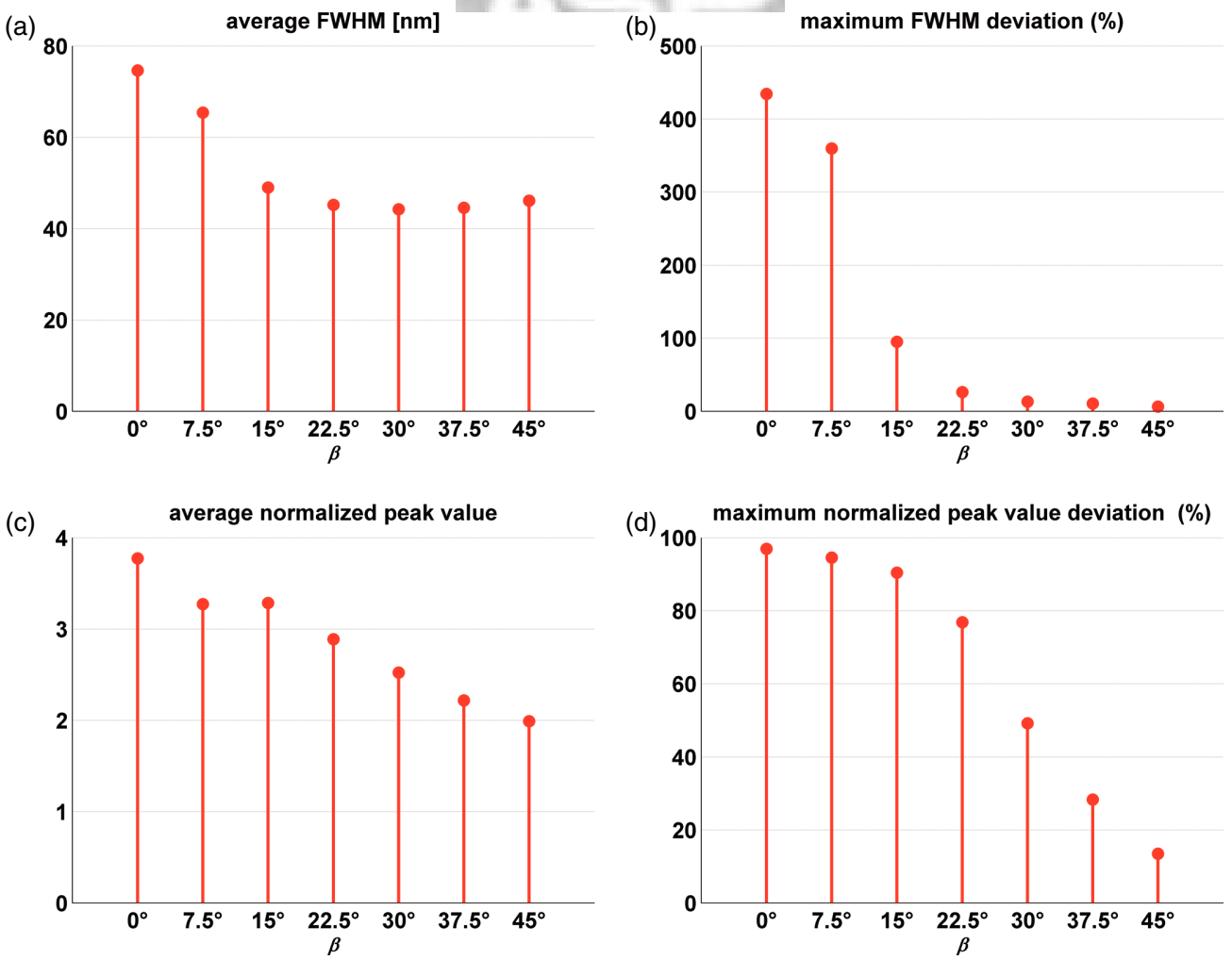

Fig. 4. Characteristics of the near field intensity distributions for the probes with corrugations arranged in a spiral-like fashion for variable shift angle $\beta\left(\gamma=160^{\circ}\right.$; radius $\left.20 \mathrm{~nm}\right)$ : (a) average FWHM; (b) maximum deviation from the average FWHM; (c) average peak value normalized to the one achieved in an axisymmetric probe under radially polarized excitation; (d) maximum deviation from the average normalized peak value. 
(Fig. 4(a)). Correspondingly, the same trend is also shown by the maximum variation in the FWHM (Fig. 4(b)). Moreover, with increasing shift angle, the deviation from the average peak value decreases as well (Fig. 4(d)), but the average peak value declines slightly (Fig. 4(c)). These results can be explained if recalling that, as $\beta$ rises, the asymmetry turns out to be more equally distributed in all spatial directions. Therefore, the behaviour over all the mutual orientations $\alpha$ becomes more uniform, which accounts for the small deviations from the average peak value and the average FWHM. However, the asymmetry along each spatial direction is weaker than the asymmetry perceived by a $x$ linearly polarized excitation when the asymmetry is fully directional with the angle bisectors of all the corrugations on the $x$ axis $\left(\beta=0^{\circ}\right)$; hence, the average peak value gets lower.

The same trends with shift angle were observed considering corrugations with the same radius $20 \mathrm{~nm}$ and a different angular extension of the corrugation $\left(\gamma=90^{\circ}\right)$, as shown in Figure 5. However the achievable peak values are lower than those retrieved with a broader extension, as will be shown also in the analysis of the impact of $\gamma$. Ma

Just as in the previous simulations, for the investigation of the effect of another geometric parameter of the corrugation, i.e., its angular extension $\gamma$, all the corrugations are supposed to be identical: the radius of each truncated toroid is $20 \mathrm{~nm}$ and the shift angle $\beta$ between consecutive corrugations amounts to $45^{\circ}$. In our calculations we varied the angular extension $\gamma$ of the corrugations from $30^{\circ}$ to $160^{\circ}$ with a step of $10^{\circ}$. Also for this set of simulations we considered all the possible mutual orientations $\alpha$ of the input linear polarization with respect to the corrugations and examined the average behaviour and the deviations from the average, as previously explained. Results are reported in Figure 6.

For angular extensions $\gamma$ above $70^{\circ}$, the field confinement under linearly polarized excitation, as measured with the FWHM, becomes remarkable compared to an axisymmetric probe (Fig. 6(a)). The stronger asymmetry for increasing extension results in higher peak values (Fig. 6(c)), but the homogeneity of the probe behaviour in all spatial directions is guaranteed for any value of $\gamma$, as demonstrated by the deviations from the average peak value, which are below 20\% (Fig. 6(d)), and those in the spot size, even under $10 \%$ for the cases of stronger confinement (Fig. 6(b)). Simulations with a different shift angle $\left(15^{\circ}\right)$ and variable angular extension have shown similar trends in terms of average FWHM and peak value, but higher deviations due to the less homogeneous distribution of the asymmetry for a smaller shift angle commented in Figures 4 and 5.

Finally, we also assessed the effect of a variation in the radius which we increased from $20 \mathrm{~nm}$ to $35 \mathrm{~nm}$ with a step of $5 \mathrm{~nm}$. The shift angle $\beta$ between corrugations
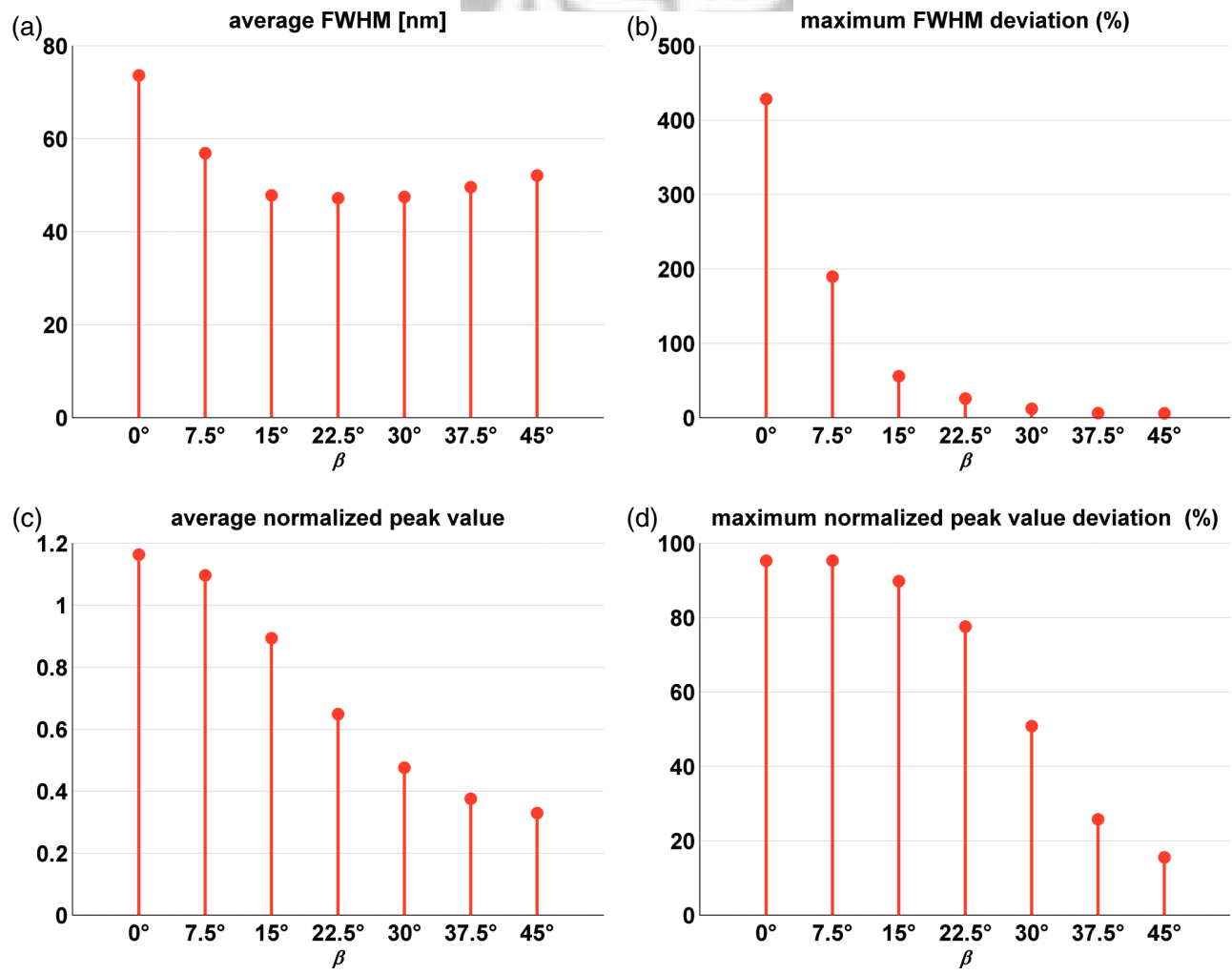

Fig. 5. Characteristics of the near field intensity distributions for the probes with corrugations arranged in a spiral-like fashion for variable shift angle $\beta\left(\gamma=90^{\circ}\right.$; radius $\left.20 \mathrm{~nm}\right)$ : (a) average FWHM; (b) maximum deviation from the average FWHM; (c) average peak value normalized to the one achieved in an axisymmetric probe under radially polarized excitation; (d) maximum deviation from the average normalized peak value. 
(a)

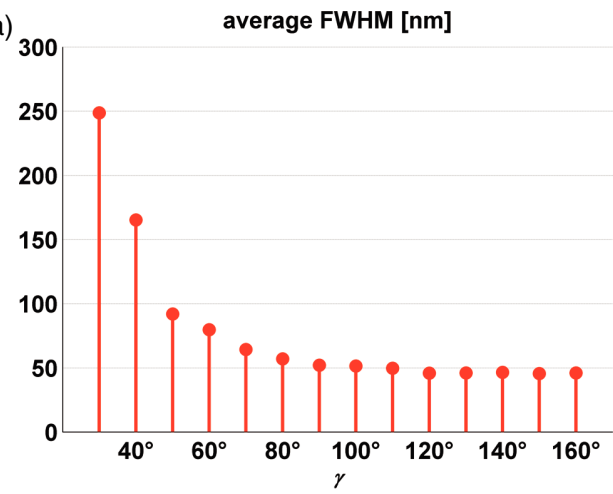

(c)

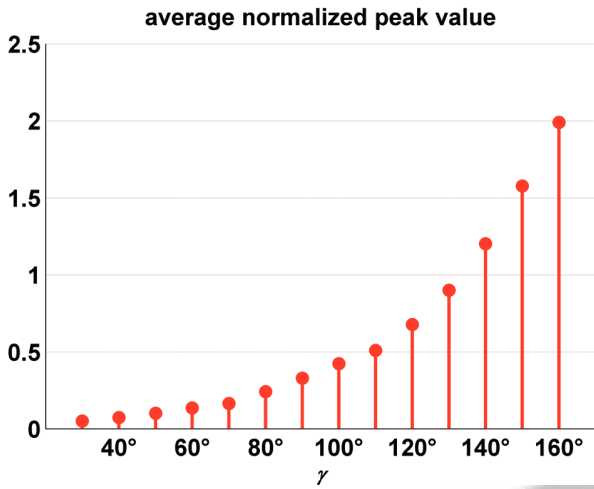

(b)

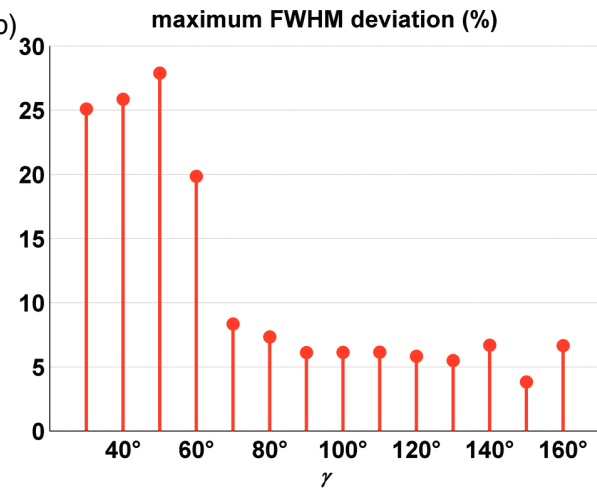

(d)

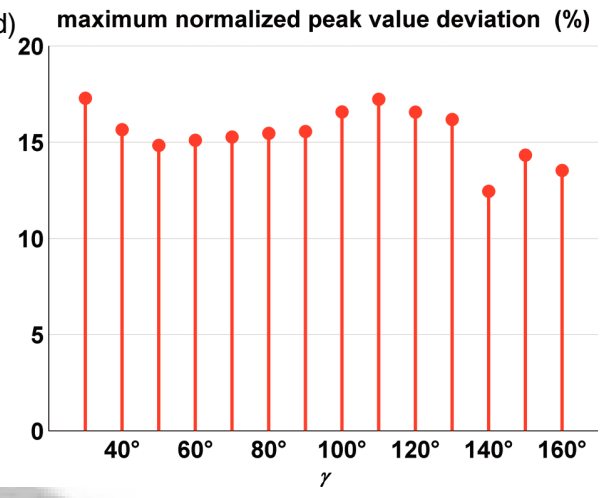

Fig. 6. Characteristics of the near field intensity distributions for the probes with corrugations arranged in a spiral-like fashion for variable angular extension $\gamma\left(\beta=45^{\circ}\right.$; radius $\left.20 \mathrm{~nm}\right)$ : (a) average FWHM; (b) maximum deviation from the average FWHM; (c) average peak value normalized to the one achieved in an axisymmetric probe under radially polarized excitation; (d) maximum deviation from the average normalized peak value.

and the angular extension of each single corrugation $\gamma$ were $45^{\circ}$ and $160 \mathrm{~nm}$, respectively. Results are plotted in Figure 7.

The graphs show that spatial localization of the field is comparable in all the cases (Figs. 7(a and b)) with variations in spot size always below 20\%; the higher variations in the peak values over all the possible mutual orientations for increasing radius (Fig. 7(d)) are compensated by an increase in average peak values (Fig. 7(c)). Similar trends were observed also for the probe with a spiral corrugation of variable radius. ${ }^{24}$

In brief, the introduction of spiral-arranged azimuthal corrugations makes the probe behaviour robust with respect to variations in the input polarization direction, as destructive interference of the SPPs excited on the opposite sides of the probe by a linearly polarized mode is avoided for any arbitrary orientation of the input linearly polarized excitation. Light funnelling to an ultrasmall spot is achieved irrespective of the input polarization direction. The reason for a superfocusing effect under linearly polarized excitation similar to the one observed in an axisymmetric probe under radially polarized excitation is the same for all the asymmetric structures based on corrugations, either directional or adirectional, and lies in the intermingling of mode coupling mechanisms between waveguide modes and SPPs and in the interaction of the SPPs on the outer metal surface with the corrugations. ${ }^{17,24}$ In fact, as explained previously, in an axisymmetric probe, the SPPs excited by a linearly polarized mode have opposite polarities on the opposite sides of the probe and therefore cancel out at the probe apex. The presence of the corrugation modifies the characteristics of the SPPs by causing the electric fields on the opposite sides of the probe not to have opposite phases any longer, hence avoiding destructive interference and allowing the achievement of field localization typical of the SPPs excited by a radially polarized mode in an axisymmetric structure. The main difference between directional and adirectional asymmetries is that, in the latter case, the extension of the asymmetry to all spatial directions allows the attainment of such effect for any linear polarization direction. A more uniform distribution of the asymmetry over all spatial directions corresponds to a more stable behaviour under diverse linear polarization directions, which means lower variations in peak value and spot size as the mutual orientation between the corrugations and the polarization direction changes. Moreover, in agreement with what has been observed for directional asymmetries, better field localization ensues from stronger asymmetries extended to larger tip sections as is the case for corrugations with broader angular extension. 
(a)

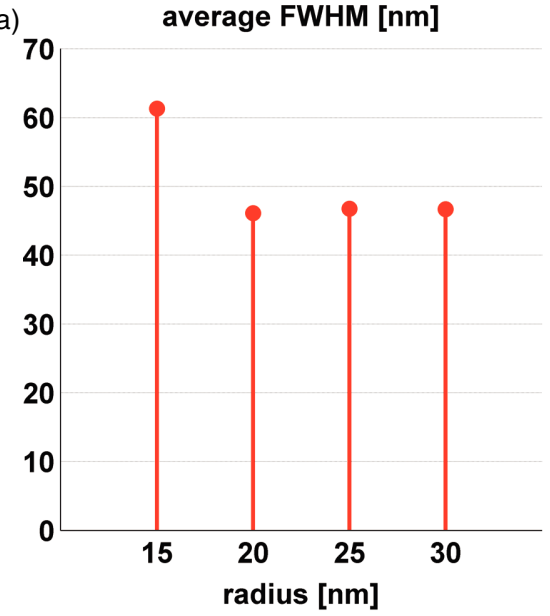

(c)

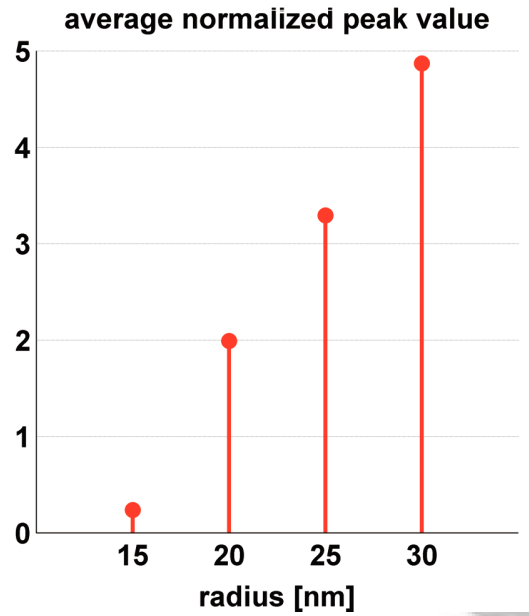

(b)

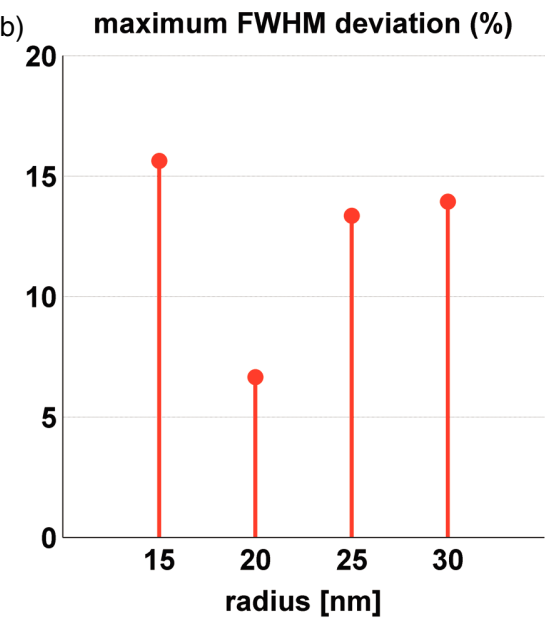

(d) maximum normalized peak value deviation (\%)

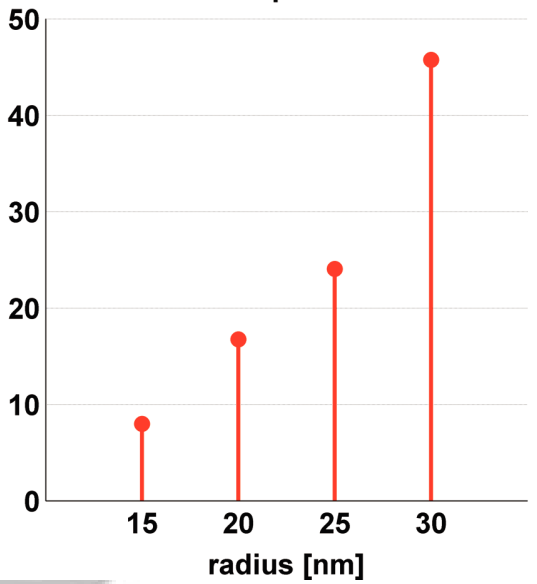

Fig. 7. Characteristics of the near field intensity distributions for the probes with corrugations arranged in a spiral-like fashion for variable radius of the corrugation $\left(\beta=45^{\circ} ; \gamma=160^{\circ}\right)$ : (a) average FWHM; (b) maximum deviation from the average FWHM; (c) average peak value normalized to the one achieved in an axisymmetric probe under radially polarized excitation; (d) maximum deviation from the average normalized peak value.

\section{CONCLUSIONS}

Our simulations have shown how simple azimuthal corrugations properly arranged in a spiral-like fashion (that is to say shifted one with respect to the other) allow the achievement of superfocusing under any arbitrarily oriented linearly polarized excitation, thereby waiving any requirement on input polarization alignment typical of asymmetric structures with directional asymmetries. In fact, in this case, the input linear polarization does not need any longer to be aligned along one specific direction, because the asymmetry is present in any spatial direction.

Geometric parameters of this novel adirectional asymmetry (like shift angle, radius and angular extension of the corrugation) can be adjusted to improve field localization and decrease its sensitivity to the input polarization direction. By properly tuning the characteristics of the asymmetry, variations in peak value and spot size lower than $20 \%$ and $10 \%$ respectively can be obtained under variable orientation of the input polarization direction with respect to the corrugation. On the contrary, if the corrugations are all aligned in one direction (zero shift angle), variations in peak value and FWHM can be as high as $100 \%$ and $450 \%$, respectively. The proposed structure can be further optimized by analysing the effect of a variation of other parameters like the number of corrugations and the period. However an accurate numerical investigation of the effect of such parameters looks challenging due to computational limits.

Another issue which deserves further investigation is the behaviour of the probe with adirectional asymmetries under circularly polarized excitation. In this case the effect of the handedness of the input excitation with respect to the handedness of the spiral-arranged corrugations could play a role and open up new applications. Such chiralitydependent behaviour has been demonstrated for 2D spiral plasmonic lenses (whose focusing properties and peak intensity could be tuned by varying the handedness of the polarization $)^{25}$ and photonic metamaterials made up of $3 \mathrm{D}$ gold helices. ${ }^{26}$

Compared to the previously proposed adirectional asymmetry based on a spiral corrugation, the current structure 
exhibits a superfocusing effect equally robust to variations in the direction of the input linearly polarized excitation, but simultaneously appears more feasible for fabrication, using for example focused ion beam milling, already employed for the fabrication of corrugations in fully metal probe structures for far field grating coupling of surface plasmons. ${ }^{27}$

Acknowledgments: The authors gratefully acknowledge the support of the Swiss National Science Foundation (project number 200221-115895).

\section{References}

1. T. J. Antosiewicz, M. Marciniak, and T. Szoplik, Photonic Crystals: Physics and Technology, edited by C. Sibilia, T. M. Benson, M. Marciniak, and T. Szoplik, Springer, Milan (2008), Chap. 11, pp. 217-238.

2. S. Mononobe, Progress in Nano-Electro-Optics III: Industrial Applications and Dynamics of the Nano-Optical System, edited by M. Ohtsu, Springer, Berlin-Heidelberg (2005), Chap. 1, pp. 1-56.

3. L. Novotny and B. Hecht, Principles of Nano-Optics, Cambridge University Press, New York (2006).

Wed, 28 Ma

4. B. Biehler and A. H. La Rosa, Rev. Sci. Instrum. 73, 3837 (2002).

5. L. Liu and S. He, Appl. Opt. 44, 3429 (2005).

6. N. M. Arslanov, J. Opt. A: Pure Appl. Opt. 8, 338 (2006).

7. M. I. Bakunov, S. B. Bodrov, and M. Hangyo, J. Appl. Phys 96, 1775 (2004).

8. T. J. Antosiewicz and T. Szoplik, Opt. Express 15, 10920 (2007)

9. T. Yatsui, M. Kourogi, and M. Ohtsu, Appl. Phys. Lett. 71, 1756 (1997).
10. Z.-B. Li, W.-Y. Zhou, W.-G. Yan, and J.-G. Tian, Plasmonics 6, 149 (2011).

11. A. Hartschuh, Angew. Chem.-Ger. Edit. 47, 8178 (2008).

12. W. Ding, S. R. Andrews, and S. A. Maier, Phys. Rev. A 75, 0638221-10 (2007).

13. A. Bouhelier, J. Renger, M. R. Beversluis, and L. Novotny, J. Microsc. 210, 220 (2003).

14. H. G. Frey, C. Bolwien, A. Brandenburg, R. Ros, and D. Anselmetti, Nanotechnology 17, 3105 (2006).

15. W. Chen and Q. Zhan, Opt. Express 15, 4106 (2007).

16. W. Nakagawa, L. Vaccaro, H. P. Herzig, and C. Hafner, J. Comput. Theor. Nanosci. 4, 1 (2007).

17. V. Lotito, U. Sennhauser, and C. Hafner, Opt. Express 18, 8722 (2010).

18. V. Lotito, U. Sennhauser, and C. Hafner, J. Comput. Theor. Nanosci. 7, 1596 (2010).

19. H. G. Frey, F. Keilmann, A. Kriele, and R. Guckenberger, Appl. Phys. Lett. 81, 5030 (2002).

20. T. H. Taminiau, F. B. Segerink, R. J. Moerland, L. K. Kuipers, and N. F. van Hulst, J. Opt. A: Pure Appl. Opt. 9, S315 (2007).

21. M. C. Quong and A. Y. Elezzabi, Opt. Express 15, 10163 (2007).

22. J. S. Lee, S. Han, J. Shirdel, S. Koo, D. Sadiq, C. Lienau, and N. Park, Opt. Express 19, 12342 (2011).

23. V. Lotito, U. Sennhauser, and C. Hafner, PIERS Online 7, 394 (2011).

24. V. Lotito, U. Sennhauser, C. Hafner, and G.-L. Bona, Plasmonics 6, 327 (2011)

25. W. Chen, D. C. Abeysinghe, R. L. Nelson, and Q. Zhan, Nano Lett. 10, 2075 (2010).

26. J. K. Gansel, M. Thiel, M. S. Rill, M. Decker, K. Bade, V. Saile, G. von Freymann, S. Linden, and M. Wegener, Science 325, 1513 (2009).

27. C. Ropers, C. C. Neacsu, M. B. Raschke, M. Albrecht, C. Lienau, and T. Elsaesser, Jpn. J. Appl. Phys. 47, 6051 (2008).

Received: 26 September 2011. Accepted: 14 October 2011. 